\title{
Applying Ensemble Neural Networks to an Inverse Problem Solution to Prosthetic Socket Pressure Measurement
}

\author{
P Davenport, S Noroozi, P Sewell \\ Faculty of Science and Technology \\ Bournemouth University \\ Poole, UK \\ PDavenport@bournemouth.ac.uk
}

\author{
S Zahedi \\ Chas A Blatchford and Sons Ltd. \\ Basingstoke, UK
}

\begin{abstract}
Ensemble neural networks are a commonly used as a method to boost performance of artificial intelligence applications. By collating the response of multiple networks with differences in composition or training and hence a range of estimation error, an overall improvement in the appraisal of new problem data can be made. In this work, artificial neural networks are used as an inverse-problem solver to calculate the internal distribution of pressures on a lower limb prosthetic socket using information on the deformation of the external surface of the device. Investigation into the impact of noise injection was studied by changing the maximum noise alteration parameter and the differences in network composition by altering the variance around this maximum noise value. Results indicate that use of ensembles of networks provides a meaningful improvement in overall performance. RMS error expressed as a percentage of the total applied load was $3.86 \%$ for the best performing ensemble, compared to $5.32 \%$ for the mean performance of the networks making up that ensemble. Although noise injection resulted in an improvement in typical network estimates of load distribution, ensembles performed better with low noise and low variance between network training patterns. These results mean that ensembles have been implemented in the research tool under development
\end{abstract}

Keywords—artificial intelligence; amputees; normal stress

\section{INTRODUCTION}

The lower-limb prosthetic socket is a crucial component in an artificial limb to ensure that an active amputee can mobilize efficiently and in comfort [1]. A wide range of techniques have been employed to evaluate the pressure distribution caused by standing and walking: however all suffer from significant limitations [2]. No systems are in routine use in rehabilitation. For this reason, an inverse problem solution has been developed. This relates strain measurements from the external surface of the socket wall to internal pressures using a neural network. Doing so maintains the integrity of the stump/socket interface, without requiring detailed knowledge of the socket geometry or tissue properties. The principle of this approach has been demonstrated previously [3], as have several techniques to improve the accuracy and reliability of the networks in use, including noise injection and via a correction

This work is sponsored by Chas A Blatchford and Sons Ltd. and an EPSRC CASE Industrial Studentship factor from mapping polynomial functions to the network response [4].

A natural extension of these measures is to incorporate ensembles of neural networks into the solution: this being a well-established technique for boosting overall performance by averaging estimates across multiple separate networks [5]. Such ensembles benefit from diversity in the constituent networks; however this property is likely problem specific. In this work, the changes in ensemble variance were systematically altered by changing the magnitude and spread of the noise injection values. The impact of this on overall performance was evaluated using a separate set of measurement cases.

\section{METHODS}

Prosthetic sockets are structures that are challenging to analyze using traditional analytical techniques. These devices are created to fit the contours of the residual limb in a manner which enables secure and consistent application of forces to the appropriate regions of the stump. This means that every socket differs in shape and size according to the specific requirements of the user and the intentions and skills of the prosthetist [6]. Individual sockets may have inhomogeneous material thickness from the manufacturing processes used. The advantage of an inverse problem approach utilizing artificial neural networks over traditional numerical analysis is that knowledge of the size, shape and thickness of the socket is not required, nor specific details of the residual limb beyond identification of regions where pressure information is valuable. 


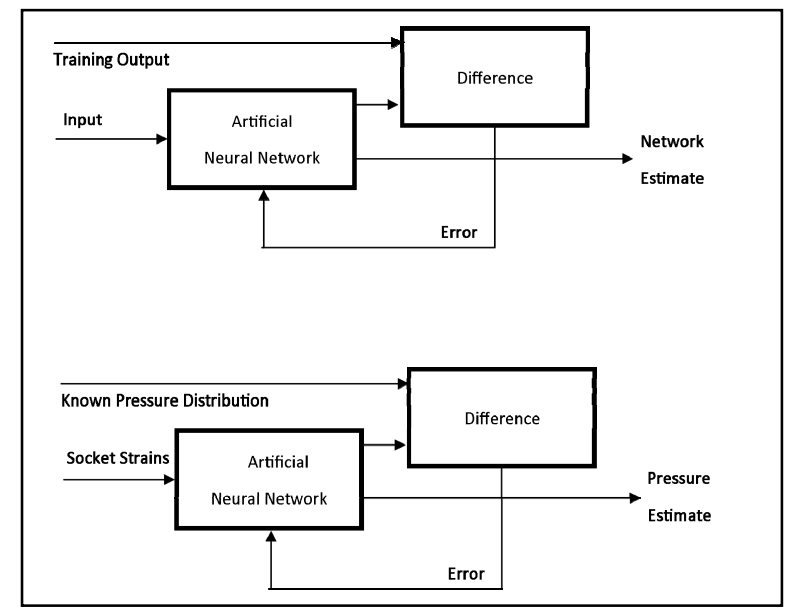

Fig.1. A representation of the neural network training process (adapted from [3]

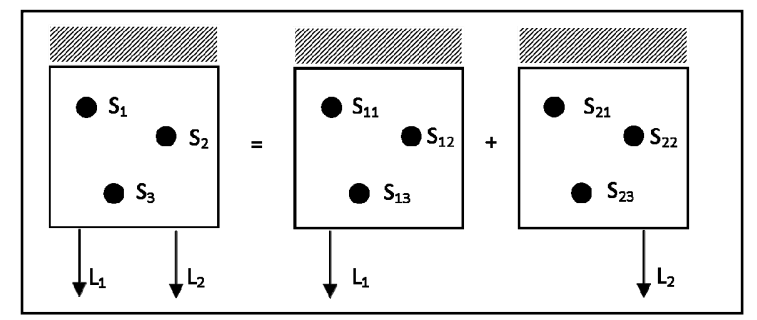

Fig. 2. The superposition principle. The effect of multiple loads (L) on a set of strain measurements (S) is equivalent to the sum of the effects of individual loads. This is a simplified two-load/three-strain system: the socket in this study used 11 strains and eight separate loading locations. This figure is adapted from [4].

Neural networks are able to identify associations - for example a multilayer perceptron is able to ascertain the nonlinear transfer functions that underpin the mechanical behavior of a system in response to changes in the input conditions [7]. A neural network can in a sense be considered a 'black box': an information processing system that receives input and produces output, and which learns the correct association between these from a training process. In this application, the network is used to take strains measured on the external socket wall as input, and the interface pressures creating these deformations as the output. Once sufficiently trained (the adjustment of the weights and biases to the internal transfer functions to minimize the error between expected and measured pressures) then the system can be supplied the strain data only, and an estimate of the pressure distribution made.

Networks of this type require many training cases to adequately create this transfer function. In order to produce sufficient training cases for the neural network to converge, two assumptions were made about the system. The first was that deformation of the socket remained linear throughout the range of loads applied. Secondly, the principle of superposition loads was utilized. This states that the effect of two loads applied simultaneously is equivalent to the sum of the effects of the constituent loads applied separately. Figure 2 shows an example of the superposition principle in effect in a two loadthree measurement system. Equation 1 is an example of a training seed file produced from such a system.

$$
\text { TrainingSeedFile }=\left[\begin{array}{cc}
S 11 & S 21 \\
S 12 & S 22 \\
S 13 & S 23 \\
L 1 & L 2
\end{array}\right]
$$

In order to produce multiple training cases covering the complete range of load distributions, a bank of random numbers between 0 and 1 are generated. A random number for each load position is scaled by the applied load value. The assumption of linearity means that the resultant strains from this single load can be calculated. The process is repeated for each other load position, and the strains summed to combine them to a single set of strain patterns with the combination of loads that would create them. The process of generating random numbers can continue indefinitely in order to create a training file of any number of cases. These load cases are supplemented by so called 'isolated' loads: simulated loads between zero and maximum in one position only in order to improve training on cases similar to this.

To improve the generalization performance of the system, noise injection was utilized. In this case, the magnitude of noise was specified as a maximum percentage of the measured relative strain. Each strain in each case was modified by either adding to or subtracting from this strain a randomly generated value up to this percentage.

For data collection the socket was mounted on a rotating platform. Loads were applied using a spring loaded arm. When restrained, the load arm can be adjusted to fit inside the socket at any desired height. The load arm, when released, applies a consistent load to a specific point on the internal socket. The contact patch of the loading device is mounted using a ball joint to ensure the force is always applied normal to the socket wall. The contact patch itself is circular and $12.5 \mathrm{~mm}$ in diameter.

A Northplex (Northsea Plastics Ltd. Glasgow, UK) transtibial check socket was instrumented with $11350 \Omega$ strain gauges superglued to the external surface. Each gauge was in a quarter-bridge configuration, and supplemented by a further strain gauge that was kept unloaded to act as temperature compensation. The gauges were distributed around the socket,

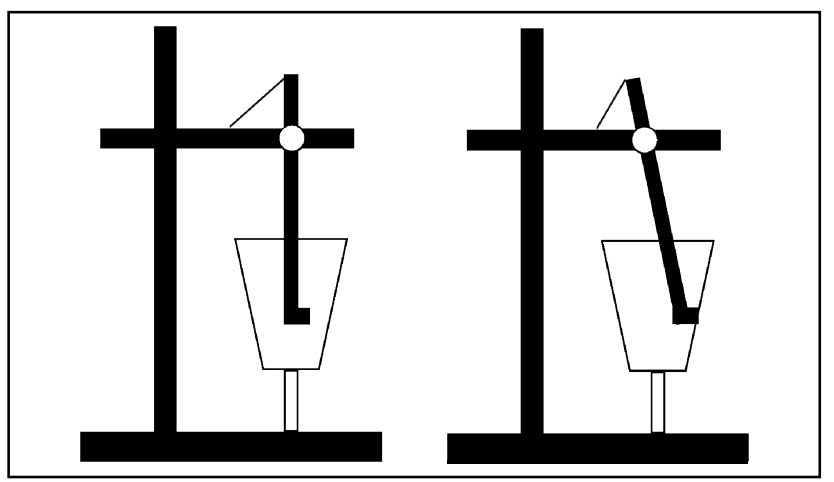

Fig.3. The function of the loading arm in use. The point of application can be moved to any height within the socket, and the socket itself rotated, so that any position on the interior surface can be loaded via the loading point. A strain gauge on the pivoting arm provides the measurement of applied load. 
in varying orientations. The values from these gauges acted as the inputs to the neural network, paired with known input forces applied to the socket wall.

The strain voltages were collected using three VLink LXRS wireless measurement nodes (Lord Microstrain Sensing Systems, Cary, North Carolina, USA), collected at $16 \mathrm{~Hz}$ using custom LabView software (National Instruments). Constant loads on internal positons were streamed to file, and modified for temperature compensation using the unloaded strain gauge and a two second moving average. Measurements were taken as the mean of values over approximately five seconds of loading.

Positions on eight internal patches were tested - two heights separated by $50 \mathrm{~mm}$ across the central area of the socket with four loads at $90^{\circ}$ intervals (supplemented by a 'neutral' recording of zero applied load to which the other positions were referenced). Using the superposition method described above, these eight loads were used to generate 1000 training cases of random combinations of load distributions. These were supplemented by 50 random magnitude 'isolated' loads for each position, for a training file of 1400 training cases in total. Details of the network design are supplied in table 1 .

Ensembles of 100 networks were created. Each ensemble differed in the magnitude and variation of the noise injection parameter. Maximum noise magnitude was centered on 0, 5, 10,15 and $20 \%$ of the applied load. Variation within the ensemble was also changed: the standard deviation of the applied noise was set to $0,1,2,5$ and 10. Therefore, 25 ensembles were generated, each with a different magnitude of noise injection and inter-network variation.

The performance of each ensemble was evaluated using a separately collected set of 1000 superposition cases (generated in the same manner as the training data, but from a distinct set of 'seed' data, and with the omission of isolated loads). The difference between each network and the ensemble as a whole to the known values of the test data, expressed as the RMS error as a percentage of the total applied load was used as to score the quality of each ensemble.

\section{RESULTS}

The mean network performance within each ensemble, expressed as percentage RMS error and the standard deviation are shown in table 1 . In table 2, the comparative results for the mean of each networks estimate across the test file (the ensemble estimate) are presented. An example of the network estimates in included in figure 4: this shows a single training case, the estimate of one $0 \%$ noise addition network and the estimate from the 0 noise/ 0 variation ensemble.

TABLE 2 - ENSEMBLE COMPONENT PERFORMANCE

\begin{tabular}{|c|c|c|c|c|c|}
\hline \multicolumn{6}{|c|}{$\begin{array}{c}\text { Mean Network Performance of ensemble constituents } \\
\text { (RMS\% (Standard Deviation)) }\end{array}$} \\
\hline \multirow[b]{2}{*}{ Noise } & \multicolumn{5}{|c|}{ Variation } \\
\hline & 0 & 1 & 2 & 5 & 10 \\
\hline 0 & $\begin{array}{c}5.44 \\
(0.91)\end{array}$ & $\begin{array}{c}5.32 \\
(0.98)\end{array}$ & $\begin{array}{c}5.16 \\
(0.81)\end{array}$ & $\begin{array}{c}5.08 \\
(0.67)\end{array}$ & $\begin{array}{c}5.12 \\
(0.60)\end{array}$ \\
\hline 5 & $\begin{array}{c}5.03 \\
(0.61)\end{array}$ & $\begin{array}{c}5.09 \\
(0.57)\end{array}$ & $\begin{array}{c}5.01 \\
(0.58)\end{array}$ & $\begin{array}{c}5.21 \\
(0.70)\end{array}$ & $\begin{array}{c}5.00 \\
(0.58)\end{array}$ \\
\hline 10 & $\begin{array}{c}5.05 \\
(0.52)\end{array}$ & $\begin{array}{c}5.11 \\
(0.66)\end{array}$ & $\begin{array}{c}4.94 \\
(0.67)\end{array}$ & $\begin{array}{c}5.05 \\
(0.54)\end{array}$ & $\begin{array}{c}4.94 \\
(0.51)\end{array}$ \\
\hline 15 & $\begin{array}{c}4.93 \\
(0.44)\end{array}$ & $\begin{array}{c}4.87 \\
(0.46)\end{array}$ & $\begin{array}{c}4.77 \\
(0.44)\end{array}$ & $\begin{array}{c}4.94 \\
(0.52)\end{array}$ & $\begin{array}{c}4.86 \\
(0.59)\end{array}$ \\
\hline 20 & $\begin{array}{c}4.71 \\
(0.40)\end{array}$ & $\begin{array}{c}4.80 \\
(0.36)\end{array}$ & $\begin{array}{c}4.76 \\
(0.36)\end{array}$ & $\begin{array}{c}4.72 \\
(0.46)\end{array}$ & $\begin{array}{c}4.75 \\
(0.60)\end{array}$ \\
\hline
\end{tabular}

TABLE 3 - ENSEMBLE PERFORMANCE

\begin{tabular}{|c|c|c|c|c|c|}
\hline \multicolumn{6}{|c|}{$\begin{array}{c}\text { Ensemble Performance } \\
\text { (RMS\% (Percentile of ensemble constituents)) } \\
\end{array}$} \\
\hline \multirow[b]{2}{*}{ Noise } & \multicolumn{5}{|c|}{$\begin{array}{ll}\text { Variation } \\
\end{array}$} \\
\hline & 0 & 1 & 2 & 5 & 10 \\
\hline 0 & $\begin{array}{l}3.90 \\
(5.9)\end{array}$ & $\begin{array}{l}3.86 \\
(4.7)\end{array}$ & $\begin{array}{l}3.94 \\
(3.8)\end{array}$ & $\begin{array}{l}4.11 \\
(2.8)\end{array}$ & $\begin{array}{l}4.32 \\
(6.5)\end{array}$ \\
\hline 5 & $\begin{array}{l}4.20 \\
(7.1) \\
\end{array}$ & $\begin{array}{l}4.33 \\
(6.7) \\
\end{array}$ & $\begin{array}{c}4.47 \\
(15.0) \\
\end{array}$ & $\begin{array}{l}4.30 \\
(5.1) \\
\end{array}$ & $\begin{array}{l}4.26 \\
(9.0) \\
\end{array}$ \\
\hline 10 & $\begin{array}{l}4.36 \\
(6.0) \\
\end{array}$ & $\begin{array}{c}4.47 \\
(12.1) \\
\end{array}$ & $\begin{array}{l}4.25 \\
(7.3) \\
\end{array}$ & $\begin{array}{c}4.42 \\
(11.0)\end{array}$ & $\begin{array}{l}4.20 \\
(5.9) \\
\end{array}$ \\
\hline 15 & $\begin{array}{c}4.4 \\
(10.2)\end{array}$ & $\begin{array}{c}4.36 \\
(12.7) \\
\end{array}$ & $\begin{array}{r}4.27 \\
(9.5) \\
\end{array}$ & $\begin{array}{c}4.42 \\
(18.0) \\
\end{array}$ & $\begin{array}{l}4.14 \\
(6.3) \\
\end{array}$ \\
\hline 20 & $\begin{array}{c}4.28 \\
(15.2) \\
\end{array}$ & $\begin{array}{c}4.37 \\
(10.6) \\
\end{array}$ & $\begin{array}{l}4.31 \\
(6.1) \\
\end{array}$ & $\begin{array}{c}4.23 \\
(11.5)\end{array}$ & $\begin{array}{c}4.18 \\
(15.2) \\
\end{array}$ \\
\hline
\end{tabular}

TABLE 1 - NEURAL NETWORK ARCHITECTURE

Parameter Network Design

Training Method Inputs (surface strains) Outputs (internal loads) Hidden Layer Neurons Overall Node Architecture Transfer Functions

Superposition Training Cases 'Isolated' Training Cases

Total Training cases

Performance Goal

Maximum epochs

Maximum validation checks

Ensemble size
Description

Feed-Forward

Backpropagation

Levenberg-Marquardt

11

16

11-16-8

Positive-Linear

Tangent-sigmoid

1000

400

1400

0.01

100

6

100 


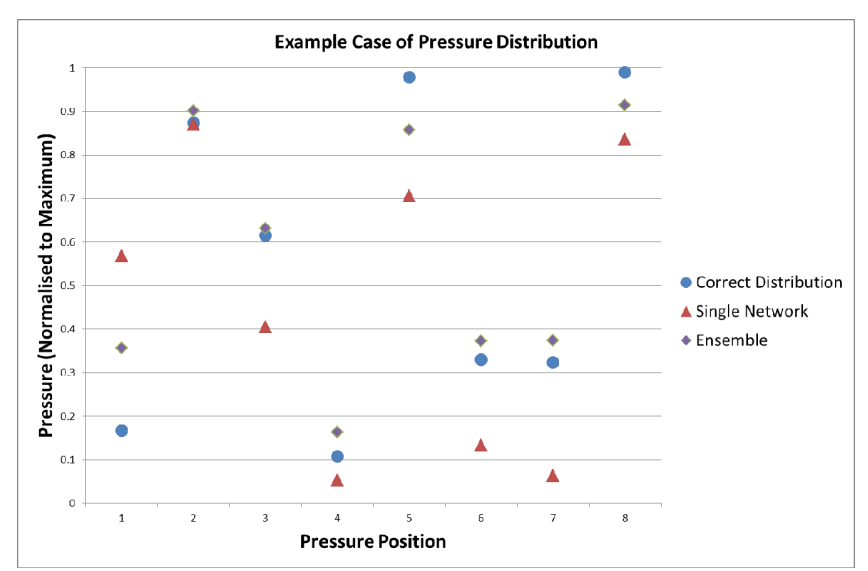

Fig.4. Example of pressure distribution estimates from one testing case. performance error of network estimates are evaluated across the range of potential inputs, a particular curve can be produced. In particular, networks tend to overestimate loads at the low end of the range and underestimate at the high end preferring to create patterns of estimation close to the center of the trained load range (Figure 4). By modelling each output channel's response to the training data and evaluating the fit of different orders of polynomial to these data points, a function that accounts for variance in network error across the range of inputs can be generated. Then, an additional term can be added to the estimation process that either adds or subtracts from each pressure estimate to better reflect the likely pressure value.

This method has been demonstrated previously in a similar system [4], and is likely to remain an effective method when utilized by an ensemble method. The ensemble estimate technique relies on the assumption that the group of networks falls symmetrically around the true answer, such that the mean estimate is close to the intended result. Although this is reliable at the center of the load range, it becomes less valid at the low or high extremes of measurement, where fewer networks will over- or under-estimate load as applicable, and a method which can correct at these locations can be expected to usefully enhance the results.

Further work required in this application is in understanding the sensitivity of the system to common perturbations in the prosthesis system - changes which have clinical or functional implications and an expected impact on the pressure distribution. An example of this is the alignment of the artificial foot to the socket. Prostheses are adjustable in translation and rotation in each plane, and for biomechanically and cosmetically acceptable gait must be adjusted in an iterative process by clinicians. Applied moments to the residual limb from loading are known to vary reliably with changes in alignments [8], although the translation from this to consistent inter-individual changes in socket pressure distribution is less clear [9], probably due to significant inhomogeneity in socket design and shape. There is potential for the introduction of personalized pattern recognition techniques using the same principles as the individual models of socket pressure measurement. Sophisticated means of utilizing and interpreting socket measurements have been only infrequently reported in the literature, and given the wealth of information that can be obtained from extended measurements of these forces, then this method can help facilitate these evaluations. However, this depends on the collection of accurate and reliable estimations, which have been enhanced by the results of this study.

\section{CONCLUSIONS}

The use of ensembles of neural networks within this application is supported by the evidence from this study. Accuracy of pressure estimates was substantially better with an ensemble over the mean performance of the ensemble constituent networks. Furthermore the chances of successfully training a better performing network were never higher than $18.0 \%$, and as low as $2.8 \%$.

One avenue for further improving the estimation is to implement a polynomial correction factor. When the 
Ensembles could be successfully trained and evaluated with minimal impact on the process of creating a solution to the problem of prosthetic socket pressure distribution estimation.

Adding noise injection as a technique tended to improve the performance of the average network created by this training process. Modifying training data by up to $20 \%$ improved mean network accuracy and reduced inter-network variance (from a standard deviation of 0.98 for the $0 \% / 1 \mathrm{SD}$ group to 0.36 for the $20 \% / 1 \mathrm{SD}$ and $20 \% / 2 \mathrm{SD}$ groups.)

A different pattern was present for the ensembles created from these groups. Here the most accurate ensembles were produced in low-noise, low variation configurations.

The results of this study inform the development of the system in use, improving the accuracy and reliability of the neural network's estimation of socket pressure, and forming the foundation for a more sophisticated method of analyzing the results of such measurements.

\section{ACKNOWLEDGMENT}

Thanks to Chas A Blatchford and Sons Ltd. for support of the lead author's PhD studies.

\section{REFERENCES}

[1] G. Pirouzi, N. Abu Osman, A. Eshraghi, S. Ali, H. Gholizadeh, W. Wan Abas, "Review of the socket design and interface pressure measurement for transtibial prosthesis," Sci. World J. p1-9 2014.

[2] P. Sewell, S. Noroozi, J. Vinney, S. Andrews, "Developments in the trans-tibial socket fitting process: a review of past and present research," Prosthet. Orthot. Int. 24 (2) p97-107 2000.

[3] R. Amali, S. Noroozi, J. Vinney, P. Sewell, S. Andrews, "Predicting interfacial loads between the prosthetic socket and residual limb for below knee amputees - a case study," Strain 42 (1) p3-10 2006.

[4] P. Sewell, S. Noroozi, J. Vinney, R. Amali, S. Andrews, "Improvements in the accuracy of an inverse problem engines output for the prediction of below-knee prosthetic socket interfacial loads," Eng Appl. Artif. Intell. 23 (6) p1000-11 2010.

[5] L. Hansen and P. Saloman, "Neural Network Ensembles," IEEE Trans. Pattern Analy. Mach. Intell. 12 (10) p993-1001 1990.

[6] S. Laing, P. Lee, J. Goh, "Engineering a trans-tibial prosthetic socket for the lower limb amputee," Ann. Acad. Med. Singapore 40 (5), p252-9 2011.

[7] M. Hagan, H. Demuth, M. Beale, Neural Network Design. Boston MA, USA, PWS Publishing, 1996.

[8] T. Kobayashi, M. Orendurff, D. Boone, "Dynamic Alignment of transtibial prostheses through visualization of socket reaction moments," Prosth. Orthot. Int. 39 (6) p512-6 2015.

[9] E.Neumann, "State of the science review of transtibial prosthesis alignment" Jour. Prosth. and Orthot. 21 (4) p175-87 2009 